\title{
Reiter's syndrome: long-term follow-up in relation to development of ankylosing spondylitis
}

\author{
A. E. GOOD
}

From the Veterans Administration Hospital, Michigan, USA

This paper will be a brief revisit to an old study of Reiter's syndrome (RS) and ankylosing spondylitis (AS) ${ }^{133},{ }^{134}$ with an attempt to bring the series up to date.

AS is defined here as back pain, persistent limitation of lumbrosacral mobility in all three planes, and grade 3 or 4 bilateral sacroiliitis (so-called New York criteria). Definite RS is defined as peripheral arthritis plus two or more of urethritis, mucocutaneous involvement, and conjunctivitis or iritis. Probable RS is peripheral arthritis and one manifestation, comprising urethritis in almost all cases.

\section{Previous study}

The previous follow-up survey ${ }^{134}$ of 35 patients two or more years beyond onset of RS showed that 10 patients followed up to 25 years had no change in radiographs of the sacroiliac joints. ${ }^{133}$ Twenty patients achieved definite changes of sacroiliitis. Nine of these also showed radiographic features in the spine pathognomonic for AS (Fig. 1). Twelve of the initial 35 patients had developed features (criteria) diagnostic for AS.

In an initial retrospective search for characteristics of prognostic value we found the following. The mean duration of disability and weight loss during the acute attacks were somewhat greater in patients who later developed sacroiliac changes. During acute attacks pain in the pelvis or low back was noted in $90 \%$ of patients who developed radiographic sacroiliitis and $70 \%$ of those who did not. At follow-up pain or stiffness of the back were reported by three-fourths of the patients with sacroiliac changes and nearly one half of those with borderline or normal sacroiliac joints. The spondylitis encountered ultimately in the 12 patients was clinically fairly typical for AS, although patients were spared severe kyphosis and involvement of the hip joints. The hypothesis was presented that ankylosing spondylitis and RS were merely separate presentations of a single underlying disease process. ${ }^{134}$

\section{Present study}

The current survey is based upon 97 patients with RS who were either encountered acutely or at routine follow-up during 1975-1978 or induced to return for the purpose of the study. The group includes 12 of the patients originally reported in 1962 or $1965 .^{133,134}$ The sample is probably skewed in the direction of severe disease, since patients with

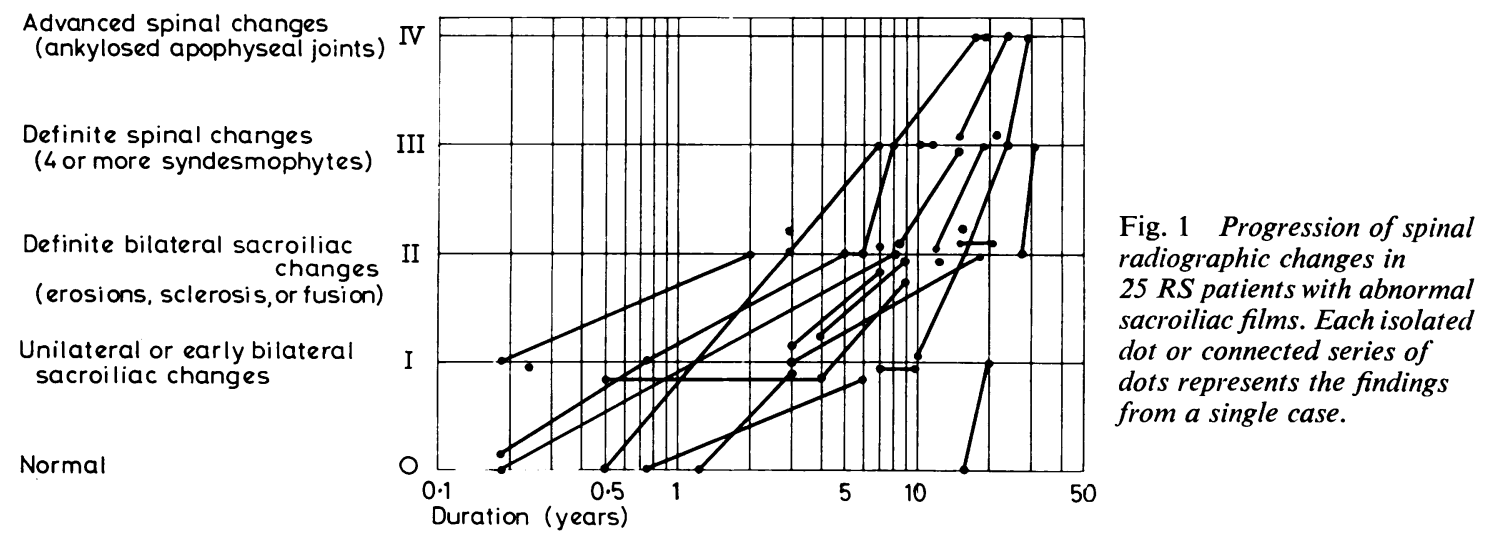




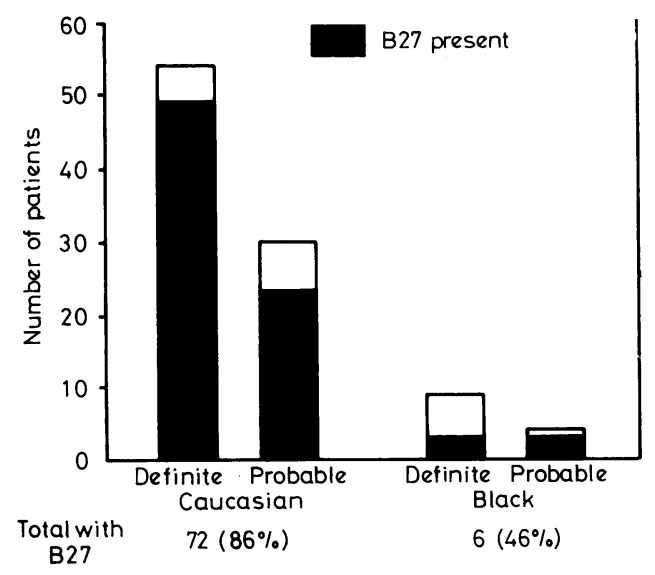

Fig. 2 Incidence of HLA-B27 among 97 patients with definite or probable $R S$ (see text for criteria).

complicated problems and multiple diagnoses tend to remain under care. We attempted zealously to call back patients who reported complete remissions by questionnaire. The series is comprised of 84 Caucasian men ( 72 definite RS, 12 probable), $86 \%$ of whom were positive for B27, and 13 black men (6 definite, 7 probable), $46 \%$ of whom were positive for HLA-B27 (Fig. 2). As with ankylosing spondylitis, ${ }^{136}$ our black patients with RS have a frequency of B27 significantly lower than that of Caucasian patients (significance level $\mathrm{P} \geqslant 0.0025$ by maximum likelihood ratio).

\section{Results}

During the initial attack of RS or the attack presenting at our medical centre $92 \%$ of patients had acute pain in the low back or pelvis compatible with pain referred from the sacroiliac joints. The pain was often present in one buttock and misinterpreted as 'hip' pain. The incidence of this acute pain was comparable in Caucasians and blacks, with and without HLA-B27.

Chronic back pain was reported by $62 \%$ overall of 78 patients seen two or more years after an acute attack. Again the incidence was not significantly different in patients with and without B27.

The most recent interpretation of sacroiliac radiographs available in 74 Caucasians are shown in Fig. 3, which relates duration of RS (defined as years since onset of peripheral arthritis) to normal and progressively abnormal films. 'Equivocal' corresponds to New York criteria grade 1 and 'definite' to grades 2 and 3 . These data show that

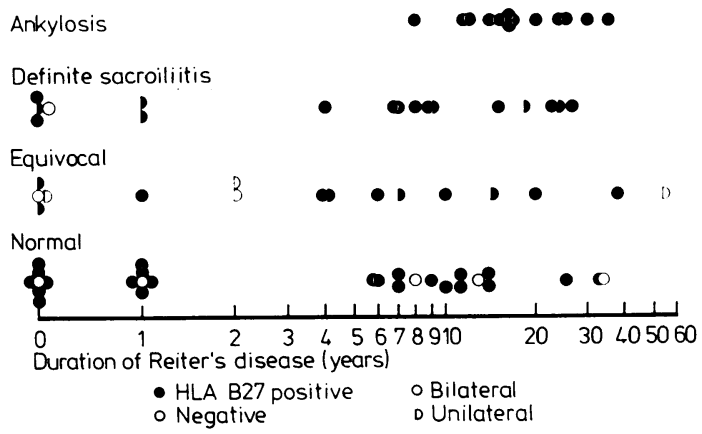

Fig. 3 Sacroiliac radiographic findings in 74 Caucasian patients.

definite sacroiliitis is nearly and ankylosis entirely limited to patients with HLA-B27. However, almost half of 45 B27-positive Caucasians followed up radiographically for two years or more have radiographs that are either normal or marginally abnormal. No definite sacroiliac disease was found among 11 black patients with available films (Fig. 4).

From the above data it is apparent that AS was found on follow-up only among Caucasians with HLA-B27, amounting to 17 cases (23\%) among 72 (Fig. 5). However, when we subtract eight patients who already had ankylosing spondylitis when first seen by us, nine $(14 \%)$ among 64 B27positive Caucasians developed spondylitis under our observation.

A computer-assisted follow-up analysis did not confirm any correlation between AS or chronic back pain with various acute features such as fever, weight loss, mucocutaneous involvement, iritis, conjunctivitis, number of attacks, and heel involvement. An analysis with the various HLA-A and B antigens disclosed a negative correlation between B35 and AS. This association reflects the frequent

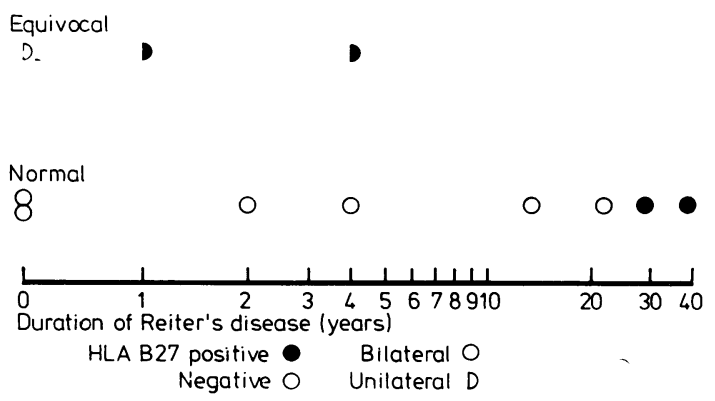

Fig. 4 Sacroiliac radiographic findings in 11 black patients. 


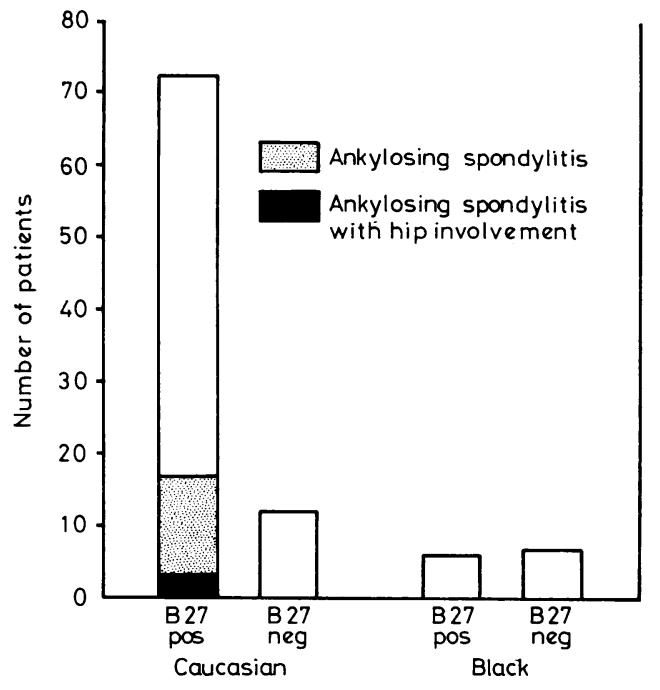

Fig. 5 Ankylosing spondylitis among 97 patients with $R S$.

appearance of B35 among Caucasians and especially blacks, who are negative for B27 and thus free from AS. Since $24 \%$ of Caucasians and $40 \%$ of blacks in our tissue-typing laboratory control series have B35, the association of B35 with RS negative for B27 did not approach statistical significance.

\section{Discussion}

From the studies of occurrence of more or less 'silent' sacroiliitis in 'healthy' people with B27, the prevalence of AS among our patients with RS is about what might be expected to accrue in longterm follow-up of any group of probands positive for the B27 antigen. ${ }^{54,64}$ Our data would support Woodrow's analysis that about $15 \%$ of men who have RS should be expected quite independently to develop AS in some degree. ${ }^{327}$

The prevalence of back pain in the longitudinal study reported here is higher than in most clinical descriptions of RS, but some other authors have emphasised back pain. Popert and coworkers in a prospective series of 82 patients with acute RS found $51(61 \%)$ with backache on entry-'one of the most constant symptoms'. Marche ${ }^{207}$ noted that sacroiliac pain was the most frequent articular manifestation during subacute or chronic RS.

During acute attacks of RS patients describe episodes of typical unilateral or bilateral sacroiliac pain, perhaps lasting one to eight weeks, and often substantiated by tenderness over the joint or pain on manoeuvres mobilising the joint. This involvement has been documented in RS by Russell et al, ${ }^{\mathbf{2 6 5}}$ who found positive sacroiliac bone scans in 23 out of $29(79 \%)$ such patients positive for HLA-B27.

It is frustrating to discuss the chronic back pain present in most patients without AS. Without a

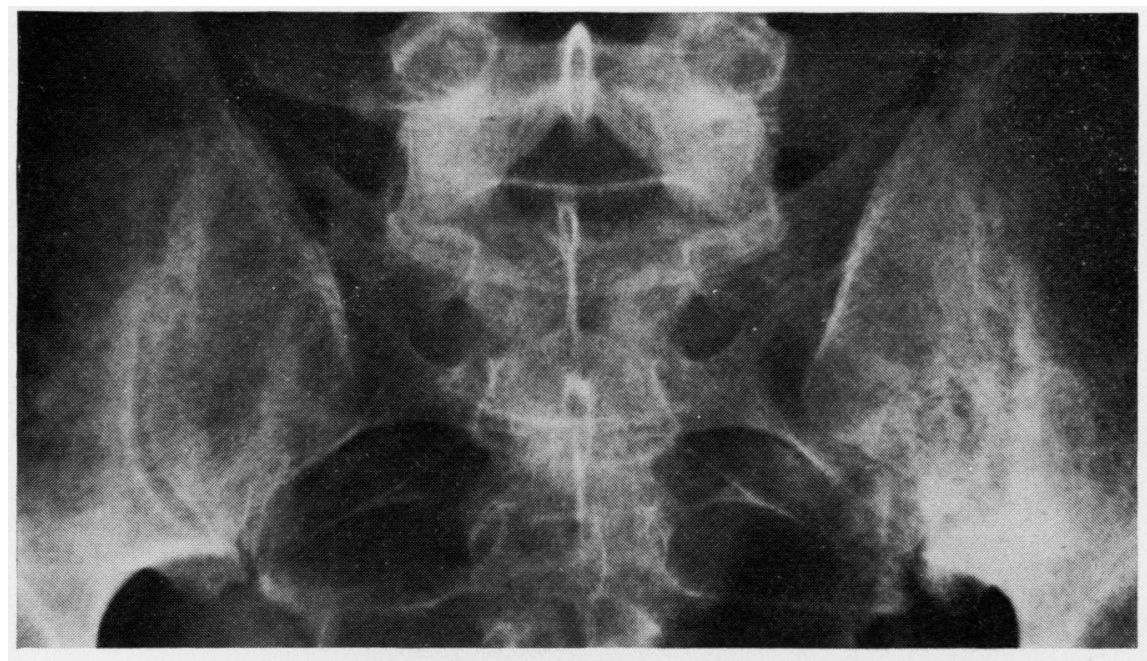

Fig. 6 Patient A. Unilateral grade 3 sacroilitis on left (left joint is on right of picture) discovered 18 years after onset of RS. Note osteophytic 'bridging' at mid-portion of right sacroiliac joint. 
longitudinal control group we must guard against unwarranted conclusions. Admittedly subjective, and often nondescript, the pain is typically worse at night or on rising but without impressive limitation of mobility and morning stiffness. The pain does not correlate significantly with radiographic sacroiliac changes, especially when we subtract those with ankylosing spondylitis.

Three patients with chronic back pain as above, all B27-positive and with known available radiographs listed as normal, were re-examined in 1978.

Patient $A$, aged 46, had a history of attacks of RS in 1957 and 1962 and normal radiographs in 1962 and 1967. On re-evaluation, films of an intravenous pyelogram of 1975 showed left sacroiliitis. Bone scan was normal. A computerised axial tomography (CAT) scan showed left sacroiliitis (Figs. 6, 7).

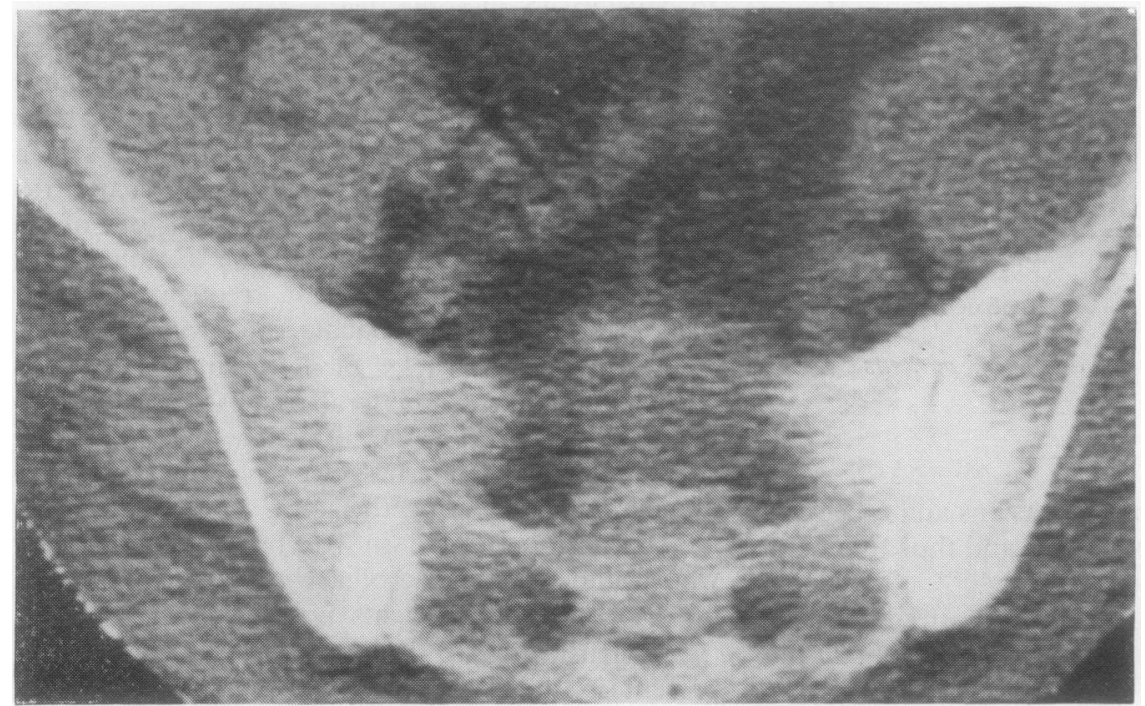

Fig. 7 Patient A. Computerised axial tomography (CAT) scan, horizontal cut at midlevel of sacroiliacs. Right joint is normal. Left joint shows erosions and extensive iliac sclerosis.

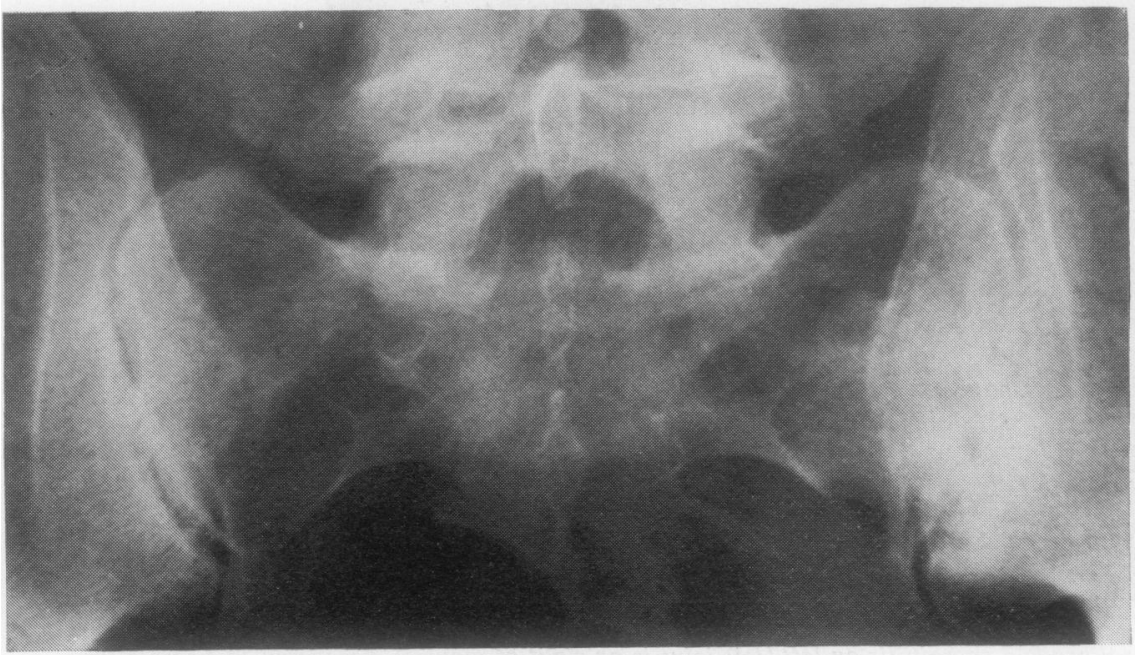

Fig. 8 Patient B. Unilateral, grade 3 sacroiliitis on left discovered 25 years after onset. 
Patient B, aged 45, had had attacks of RS in 1953 and 1959, intermittent lumbar pain since 1959 , and normal radiographs in 1959, 1961, and 1968. New radiographs showed unilateral sacroilliitis. A bone scan and CAT scan revealed bilateral sacroiliitis (Figs. 8, 9).
Patient C, aged 58, had had a prolonged attack of RS in 1962-3, chronic back pain since, and repeatedly negative sacroiliac $x$-ray films, including those of 1978. CAT scan showed bilateral, moderate sclerosis of the iliac side of the sacroiliac joints and possible erosions. Bone scans were not done. (Figs. 10, 11).

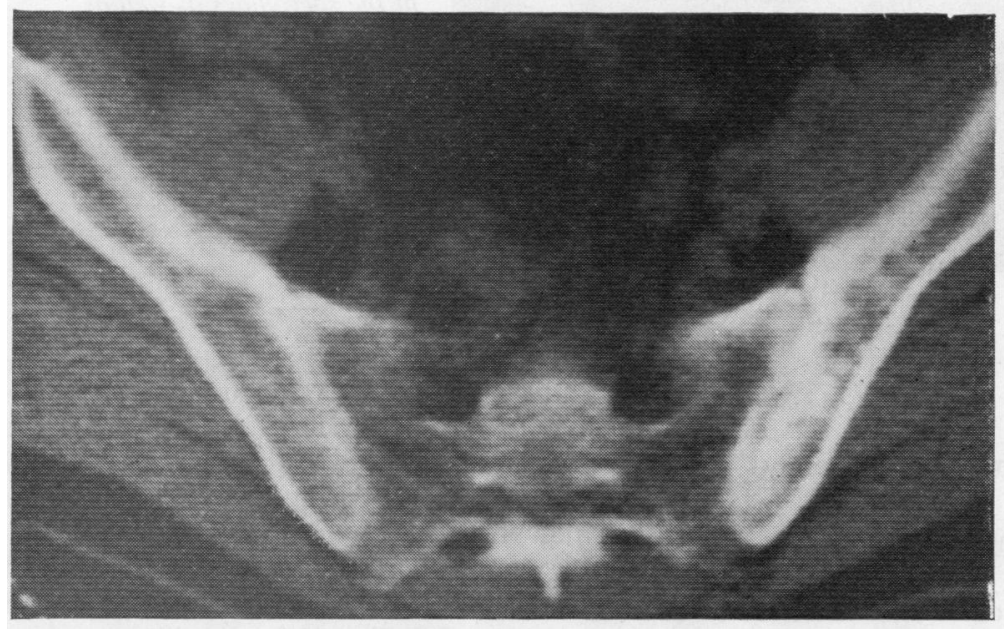

Fig. 9 Patient B. Concurrent CAT scan at midlevel showing bilateral erosive changes and predominantly left-sided sclerosis.

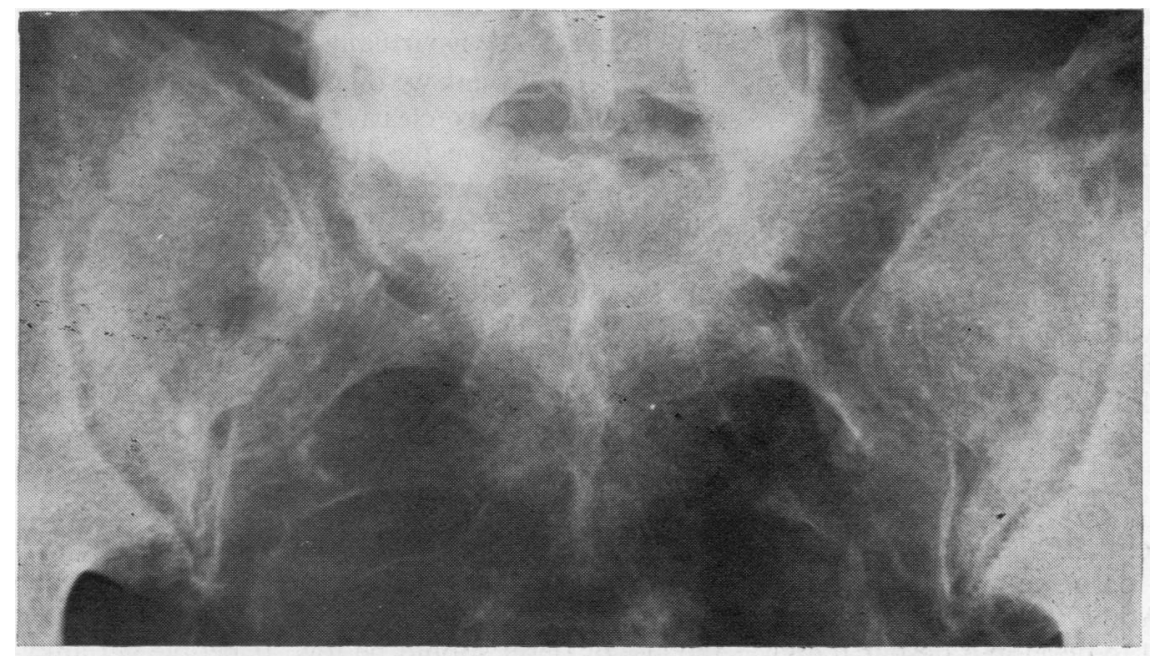

Fig. 10 Patient C. Normal sacroiliacs (or very mild left-sided sacroiliitis) 18 years after onset. 


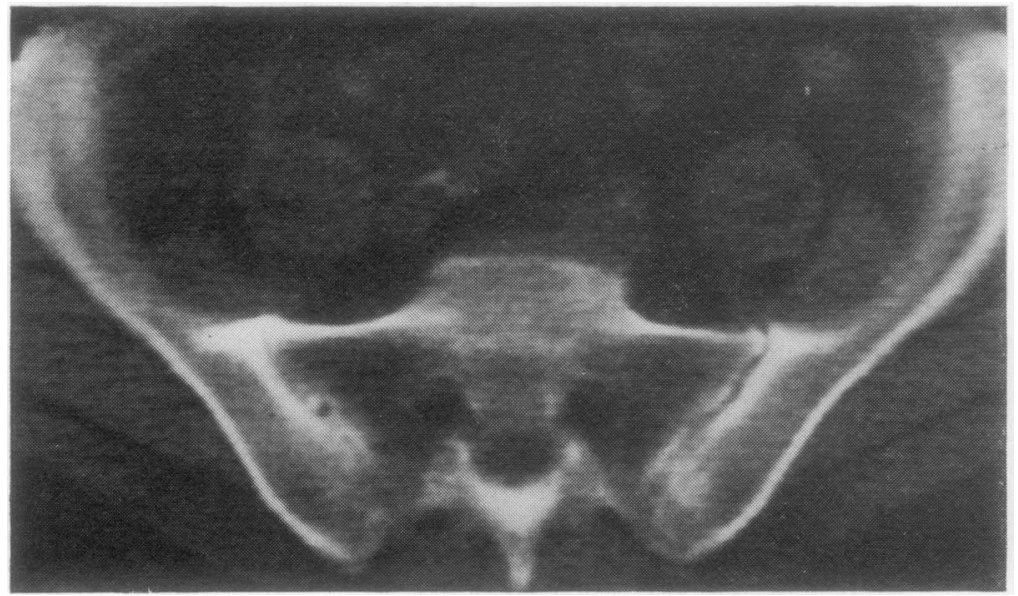

Fig. 11 Patient C. Concurrent CAT scan at midlevel showing bilateral mild sclerosis on iliac side of the sacroiliacs as well as probable erosion of right joint.

\section{Conclusion}

In summary, there may be two types of pelvispondylitic involvement with $\mathbf{R S}$.

Type I-typical aggressive ankylosing spondylitis which will coexist, perhaps independently, in about $15 \%$ of any unbiased series of B27-positive patients with RS and which rarely, if ever, occurs, in patients negative for B27.

Type II-a 'Reiter's' pelvispondylitis that differs from ankylosing spondylitis in several respects. The sacroiliac disease proceeds at low ebb. Radiographs may remain normal for years after onset of pain. The mobility of the spine and posture are not greatly impaired. Unilateral sacroiliitis is not unusual, and marked asymmetry may persist for years, possibly indefinitely.

However, definite radiographic sacroiliitis, whether unilateral or bilateral, similar to type $I$ is virtually limited to B27-positive patients. The significance and specificity of back pain in RS have not been well studied. Neither acute pain (usually pelvic) nor chronic pain correlates with B27. Chronic pain does not correlate with radiographic sacroiliitis if patients with ankylosing spondylitis are excluded.

\section{General discussion}

PROF. B. AMOR: In Algeria RS is common among newly arrived French or American soldiers. In contrast, French and Arabs who have lived there for many years seem not to develop it despite a high prevalence of both B27 and ankylosing spondylitis (AS)-perhaps higher even than in Europe. Thus it seems unlikely that AS should be a late complication of RS. Both RS and AS seem to need the same gene. This gene is probably triggered differently by some agent met fortuitously at adult age or during infancy in the country where it is endemico One could postulate, for example, the same agent. met early in life would trigger the development of AS as opposed to RS. This is further illustrated by Algerian adolescents emigrating to France, where they tend to develop RS even more than AS.

DR. A. CALIN: If we look at communities where both ankylosing spondylitis and B27 are very common (for example, the Haida Indians of British Columbia and the Pima Indians of Arizona) surprisingly Reiter's syndrome is virtually unknown.

PROF. M. ZIFF: I think we ought to get the facts about the Haida Indians clearly established. They are about $50 \%$ positive for HLA-B27. Is it true that they have a very low incidence of Reiter's syndrome? PROF. D. FORD: Yes, it seems so, but it is difficult to get data on Reiter's syndrome, since history taking isn't easy there. The incidence of ankylosing spondylitis is higher there than in any population reported so far, but despite regular consulting I don't seem to see any patients with Reiter's.

DR. CALIN: We have looked obsessionally at the Pima as a research project, and I have not yet found any RS amongst Pima Indians.

PROF. ZIFF: Are there any thoughts about that?

DR. E. ALBERT: In populations where ankylosing spondylitis is very common one would expect that the age of onset should be low. If people get ankylosing spondylitis at the age of 10 they would not have a chance to develop Reiter's disease. 
PROF. AMOR: Yes: in our department we receive many such patients with juvenile ankylosing spondylitis from Algeria. In most the disease had begun in Algeria at the age of 8 or 10 years. It is a very severe AS with hip involvement occasionally requiring complete hip prosthesis. Such a severe, young onset of AS is rarely seen in ethnically Algerian patients born in France.

DR. J. C. WOODRow: Firstly, what is the B27 prevalence in the indigenous Algerian population? Secondly, do you think that the agent which may cause Reiter's syndrome may also be one of the agents which causes some cases of ankylosing spondylitis?

PROF. AMOR: I don't know. In France we usually see posturethritis RS. This is not so in Algeria and the agent responsible for $\mathrm{RS}$ there has yet to be identified.

DR. CALIN: I am puzzled. I can't believe that the 'precipitating agent' doesn't get there. One could imagine that Shigella or Yersinia or whatever might not reach the Queen Charlotte Islands, but since the Pima Indians live within $\mathbf{4 0}$ miles of Phoenix it is highly unlikely that they are sheltered from the relevant infectious agents. The explanation for the lack of RS among the Pima would seem to be a genetic or immunological one rather than environmental.

PROF. FORD: The Haida are not necessarily sheltered either: Although we have not found any serological evidence of Yersinia infection (among the many samples of serum sent us for a study that we will mention later), we have data indicating an increased prevalence of antibodies against ureaplasmas ( $\mathrm{T}$ strain mycoplasma) in sera from the Haida Indian population. So the apparent absence of Reiter's disease remains mysterious.
PROF. A. S. RUSSELL: In the North-West territories of Canada there is a lot of Shigella, both sonnei and flexneri, and there is a high incidence of B27 in the natives. We see ankylosing spondylitis, we actually see some Reiter's disease apparently of venereal origin, but we have very rarely seen postdiarrhoeal Reiter's, and the few we did see had anti-yersinia antibodies rather than a recent bout of anti-shigella immunity. One explanation of Dr. Amor's challenging observations could be that RS is rarely attributable to an endemic agent (such as shigella), whereas the same agent appearing briskly as an epidemic is able to trigger the disease.

DR. ALBERT: I had not heard about populations with great prevalence of AS and virtual absence of RS, but I find those observations most exciting. If such striking differences were to be confirmed we might have a similar situation as we have for Epstein-Barr (EB) virus infection. As you know, in our European populations this type of infection appears as another clinical picture of infectious mononucleosis. In Africa it causes Burkitts' lymphoma, and it produces nasopharyngeal carcinoma in China. Now if one transfers people from Africa, particularly from East Africa to the United States, as has happened in history, these people once in the United States do not -as I would have expected-develop Burkitt's lymphoma. Despite their genetic background they develop infectious mononucleosis just as the white population does. If one transfers Chinese from China into Europe they will no longer get nasopharyngeal carcinoma but rather infectious mononucleosis, just as we do. That used against a genetic determination of disease expression in EB virus infection would be more consistent with my speculations that in endemic situations ankylosing spondylitis should appear at an earlier age. 